\title{
Fabry disease and COVID-19: international expert recommendations for management based on
}

\section{real-world experience}

\author{
Dawn A. Laney ${ }^{1, *}$, Dominique P. Germain ${ }^{2, *}$, João Paulo Oliveira ${ }^{3, *}$, \\ Alessandro P. Burlina ${ }^{4}$, Gustavo Horacio Cabrera ${ }^{5}$, Geu-Ru Hong (1) ${ }^{6}$, \\ Robert J. Hopkin ${ }^{7}$, Dau-Ming Niu ${ }^{8}$, Mark Thomas ${ }^{9}$, Hernán Trimarchi (1) ${ }^{10}$, \\ William R. Wilcox ${ }^{1}$, Juan Manuel Politei ${ }^{11}$ and Alberto Ortiz ${ }^{12}$
}

${ }^{1}$ Division of Medical Genetics, Department of Human Genetics, Emory University School of Medicine, Atlanta, GA, USA, ${ }^{2}$ Division of Medical Genetics, University of Versailles, AP-HP Paris Saclay University, Paris, France, ${ }^{3}$ Centro Hospitalar Universitário de São João \& Faculdade de Medicina da Universidade do Porto, Porto, Portugal, ${ }^{4}$ Neurology Unit, St Bassiano Hospital, Bassano del Grappa, Italy, ${ }^{5}$ Santa Maria de la Salud, San Isidro, Provincia de Buenos Aires, Argentina, ${ }^{6}$ Department of Cardiology, Yonsei University Severance Hospital, Seoul, Korea, ${ }^{7}$ Division of Human Genetics, Cincinnati Children's Hospital Medical Center, and Department of Pediatrics, University of Cincinnati College of Medicine, Cincinnati, OH, USA, ${ }^{8}$ Department of Pediatrics, Taipei Veterans General Hospital, Taipei, Taiwan, ${ }^{9}$ Department of Nephrology, Royal Perth Hospital, Perth, Australia, ${ }^{10}$ Nephrology service, British Hospital, Buenos Aires, Argentina, ${ }^{11}$ Department of Neurology, Fundacion Para el Estudio de Enfermedades Neurometabolicas (FESEN), Buenos Aires, Argentina and ${ }^{12}$ Unidad de Dialisis, IIS-Fundacion Jimenez Diaz, School of Medicine, UAM, IRSIN and REDINREN, Madrid, Spain

*These authors contributed equally to this work.

Correspondence to: Dawn A. Laney; E-mail: dawn.laney@emory.edu

\begin{abstract}
The rapid spread of coronavirus disease 2019 (COVID-19) caused by severe acute respiratory syndrome coronavirus 2 has raised questions about Fabry disease (FD) as an independent risk factor for severe COVID-19 symptoms. Available realworld data on 22 patients from an international group of healthcare providers reveals that most patients with FD experience mild-to-moderate COVID-19 symptoms with an additional complication of Fabry pain crises and transient worsening of kidney function in some cases; however, two patients over the age of 55 years with renal or cardiac disease experienced critical COVID-19 complications. These outcomes support the theory that pre-existent tissue injury and inflammation may predispose patients with more advanced FD to a more severe course of COVID-19, while less advanced FD patients do not appear to be more susceptible than the general population. Given these observed risk factors, it is best to reinforce all recommended safety precautions for individuals with advanced FD. Diagnosis of FD should not preclude
\end{abstract}


providing full therapeutic and organ support as needed for patients with FD and severe or critical COVID-19, although a FD-specific safety profile review should always be conducted prior to initiating COVID-19-specific therapies. Continued specific FD therapy with enzyme replacement therapy, chaperone therapy, dialysis, renin-angiotensin blockers or participation to clinical trials during the pandemic is recommended as FD progression will only increase susceptibility to infection. In order to compile outcome data and inform best practices, an international registry for patients affected by Fabry and infected by COVID-19 should be established.

Keywords: chloroquine, COVID-19, enzyme replacement therapy, Fabry disease, lysosome, pathogenesis, prevention, SARS-CoV-2

\section{INTRODUCTION}

Fabry disease (FD; OMIM \#301500) is a progressive, multisystemic, $\mathrm{X}$-linked genetic disease caused by pathogenic variants in the GLA gene encoding the $\alpha$-galactosidase enzyme [1]. Deficient $\alpha$-galactosidase enzyme activity leads to progressive storage of glycosphingolipids, particularly globotriaosylceramide $\left(\mathrm{Gb}_{3} / \mathrm{GL}_{3}\right)$ and its deacylated derivative globotriaosylsphingosine (lysoGb $\mathrm{G}_{3}$ lysoGL $L_{3}$ ), resulting in a cascade of pathogenic effects including cellular dysfunction and demise, vascular injury, inflammation and tissue fibrosis. When $\alpha$-galactosidase activity is severely deficient, it results in classic FD, a childhood-onset multisystemic disorder with reduced life expectancy in untreated patients, owing to cardiac complications, end-stage renal disease and stroke [1]. Nonclassic or later-onset FD arises in patients with some residual enzyme activity; it is still life-impacting but is characterized by a more variable disease course, in which onset is postchildhood and the main disease manifestations may be limited to a single organ [2, 3].

The rapid spread of coronavirus disease 2019 (COVID-19) in 2020 caused by the severe acute respiratory syndrome coronavirus 2 (SARS-CoV-2) has raised concerns about the possible increased risk for severe COVID-19 complications in patients with FD and the adverse effects of drugs used for COVID-19 on patients with $\mathrm{FD}$, and speculations on the interaction of drugs used for FD with COVID-19. The purpose of this article is to report the experience of an international group of experts on outcomes and provision of medical care to patients with FD during the first wave of the COVID-19 pandemic, and to use these realworld data to give consensus recommendations for the management and treatment of patients with FD during the forthcoming waves of the COVID-19 pandemic [see, Box 1].

\section{CLINICAL SPECTRUM OF COVID-19}

The clinical spectrum of COVID-19 is extremely variable ranging from completely asymptomatic or flu-like symptoms to lethal pneumonia, sepsis and multi-organ failure including respiratory failure, heart failure, acute renal injury and coagulopathy. The World Health Organization (WHO) categorizes symptoms into disease severity categories of mild, moderate, severe and critical [4] (Table 1). The calculated incidence of each category depends on local testing policies. When all exposed individuals are tested, irrespective of clinical symptoms, the case and infection fatality rates have been estimated around 2.6\% [95\% confidence interval (CI) 0.89-6.7] and 1.3\% (95\% CI 0.38-3.6), with asymptomatic infections occurring in around $45 \%$ of infected individuals, more frequently in the young and in females [5-7]. Mortality may increase in high-risk individuals, when no advanced healthcare is provided or when healthcare services are saturated, limiting access to organ support systems.

\section{RISK FACTORS FOR CRITICAL COVID-19 DISEASE}

Risk factors for critical COVID-19 disease identified in the general population include older age, male sex and the presence of comorbidities such as obesity, hypertension, diabetes, chronic kidney disease (CKD), cardiovascular disease, chronic lung disease, cancer, neurological conditions, immunocompromising conditions and smoking (Supplementary data, Table S1) [8-13]. Some of these risk factors overlap with symptoms seen in adult patients with FD including cardiac complications, renal disease, pulmonary disease and strokes (Figure 1).

\section{RATIONAL FOR THE CONCERNS REGARDING COVID-19 IN PATIENTS WITH FD: POTENTIAL OVERLAP BETWEEN THE RESPECTIVE PATHOGENESIS AND TARGET ORGANS}

Four key features contributed to the severe pathogenesis of COVID-19 [14]. First, cellular infection is dependent on a highlevel expression of angiotensin-converting enzyme 2 (ACE2) at the plasma membrane, as found in type II pneumocytes, endothelial cells, tubular epithelial cells, and cardiac and gastrointestinal cells, which may explain the multi-organ dysfunction [15-19]. Second, the inflammatory response may be extremely severe, with an excessive release of cytokines ('cytokine storm'), causing endothelial and organ injury by itself. Third, a prothrombotic state driven by inflammation and endothelial cell injury, which may lead to micro- or macrothrombosis, including pulmonary thromboembolism, myocardial infarction and stroke. Fourth, hypoxemia due to lung involvement and hemodynamic instability are thought to contribute to tissular injury during COVID-19 (Supplementary data, Figure S1B).

The pathogenesis and target organ injury of COVID-19 are common to or partly overlap with those of FD (Figure 1), raising theoretical concerns that the pre-existing FD pathology may serve as a 'first hit' that increases patients' risk for serious complications when infected by COVID-19.

Specifically, endothelial cell $\mathrm{Gb}_{3} / \mathrm{GL}_{3}$ storage is a hallmark of FD, and endothelial dysfunction and thrombophilia have been proposed among the mechanisms underlying vascular involvement in patients with classic FD [20]. Plasma levels of soluble vascular cell adhesion molecule-1 (VCAM-1), a cell adhesion molecule that contributes to leukocyte recruitment into vessel walls, are significantly elevated in FD [20], and the VCAM1 gene is overexpressed in COVID-19 lungs [21]. Since COVID-19 results in distinctive vascular features in the lungs, consisting of severe endothelial injury associated with the presence of intracellular virus, disrupted cell membranes, widespread thrombosis with microangiopathy and increased angiogenesis, classically 
Table 1. WHO COVID-19 disease severity categories

\begin{tabular}{|c|c|c|c|}
\hline Severity category & Key feature & Age group & Description \\
\hline Mild disease & N/A & All ages & Symptomatic patients without evidence of viral pneumonia or hypoxia \\
\hline Moderate disease & Pneumonia & All ages & $\begin{array}{l}\text { Clinical signs of nonsevere pneumonia without features of severe } \\
\text { pneumonia }\end{array}$ \\
\hline \multirow[t]{2}{*}{ Severe disease } & Severe pneumonia & Pediatric & $\begin{array}{l}\text { Clinical signs of pneumonia plus one of the following: } \\
\text { - Central cyanosis or } \mathrm{SpO}_{2}<90 \% \text {, } \\
\text { - Severe respiratory distress } \\
\text { - General danger signs (inability to breastfeed or drink; lethargy, } \\
\text { unconsciousness or convulsions) OR fast breathing (in breaths/min) }\end{array}$ \\
\hline & & Adolescent/adult & $\begin{array}{l}\text { Clinical signs of pneumonia plus one of the following: } \\
\text { - Respiratory rate }>30 \text { breaths/min } \\
\text { - } \mathrm{SpO}_{2}<90 \% \text { on room air } \\
\text { - Severe respiratory distress }\end{array}$ \\
\hline \multirow[t]{5}{*}{ Critical disease } & $\begin{array}{l}\text { Acute respiratory } \\
\text { distress syndrome }\end{array}$ & All ages & $\begin{array}{l}\text { Onset within } 1 \text { week of known clinical insult or worsening respiratory } \\
\text { symptoms not explained by cardiac failure or fluid overload }\end{array}$ \\
\hline & Sepsis & Pediatric & $\begin{array}{l}\text { Symptoms and or signs of COVID-19 infection and }>2 \text { age-based SIRS criterion } \\
\text { of which one must be abnormal temperature or white blood cell count }\end{array}$ \\
\hline & & Adolescent/adult & $\begin{array}{l}\text { Acute life-threatening organ dysfunction caused by a dysregulated host immune } \\
\text { response to suspected or proven COVID-19 infection }\end{array}$ \\
\hline & Septic shock & Pediatric & $\begin{array}{l}\text { Any hypotension or two or three of the following: } \\
\text { - Altered mental status } \\
\text { - Bradycardia or tachycardia } \\
\text { - Prolonged capillary refill or weak pulse } \\
\text { - Fast breathing } \\
\text { - Mottled or cool skin or petechial or purpuric rash } \\
\text { - High lactate } \\
\text { - Reduced urine output } \\
\text { - Hyperthermia } \\
\text { Hypothermia }\end{array}$ \\
\hline & & Adolescent/adult & $\begin{array}{l}\text { Persistent hypotension despite volume resuscitation, requiring } \\
\text { vasopressors to maintain MAP }>66 \mathrm{mmHg} \text { and serum } \\
\text { lactate level }>2 \mathrm{mmol} / \mathrm{L}\end{array}$ \\
\hline
\end{tabular}

SIRS, systemic inflammatory response syndrome; MAP, mean arterial pressure.

affected patients with FD may be at particularly high risk for the vascular COVID-19 complications [20-22].

\section{Assessing the risk of severe COVID-19 in patients with FD}

Combining real-world data with the known pathogenesis of FD and the general population risk factors for severe complications from COVID-19 provides a window into the incidence and impact in this population. In the experience of the French national referral center for FD, 9 out of 217 patients (4.2\%) were infected by SARS-CoV-2, including 4 during the first wave (March to May 2020), and recovered without any specific treatment except for the most advanced patient who required 1-week hospitalization and oxygen during transient deterioration of his kidney function. A published case series from Italy found that none of their 129 patients with FD were diagnosed with COVID-19, although three patients did have flu-like symptoms and self-quarantined [23]. The majority of treated patients in Italy continued their enzyme replacement therapy (ERT) with the only exceptions being three individuals that refused to receive home infusions [23]. The heterogeneity in clinical presentation among patients with FD was reminiscent of that observed in the general population. This information suggests that the risk for life-threatening complications depends on the progression of FD in the individual patient. Accordingly, the specific discussion and data in this article are divided into two groups, uncomplicated FD (those without organ disease progression) and advanced FD to compare theoretical risk on real-life impact (Table 2 and Figure 2). In this publication, we report data on 22 patients with FD to provide real-world experience with COVID-19 infection in patients with FD (Table 3).

The available international dataset includes 15 patients with uncomplicated FD and 7 patients with advanced FD (Table 3).

Patients with uncomplicated FD. While FD is primarily a genetic lysosomal storage disease, an inflammatory component has also been proposed on the basis of increased inflammatory markers possibly contributing to organ damage and loss of function [24]. To date, there are no studies of COVID-19 in patients with $\mathrm{FD}$, but the overlap in impacted organ systems and the involvement of inflammation may place patients with FD at higher risk for complications compared with age- and sexmatched people who do not have FD. This is of particular concern for risk of acute deterioration in cardiac function, stroke risk, acute kidney injury and possibly the cytokine storm [25].

Some symptoms of uncomplicated FD may be exacerbated by COVID-19. A hallmark of classic FD is increased burning neuropathic pain seen prior to and during illness and fevers $[3,26$, 27]. In those experiencing this feature, infection with SARS- 
Fabry disease

Severe in males

Lysosomal undegraded substrate accumulation a-galactosidase deficiency

\section{Stroke}

Potentially severe heart and cardiovascular disease; potentially moderate lung disease

Potentially severe kidney disease

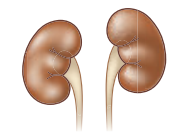

Endothelial dysfunction

Systemic inflammation

Gastrointestinal and skin disease

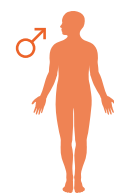

COVID-19

More severe in males

Lysosomal lumen acid $\mathrm{pH}$ promotes fusion of the endosome membrane with the virus envelope, releasing the nucleocapsid into the cytoplasm

COVID-19 drugs (e.g. chloroquine/ hydroxychloroquine) cause lysosomal injury

\section{Stroke}

Pre-existing cardiovascular and lung disease increase severity

Causes potentially lethal cardiovascular and lung disease

COVID-19 drugs (e.g. chloroquine/ hydroxychloroquine) may cause severe arrhythmia

Pre-existing kidney disease increases severity Potentially severe kidney disease

Endothelial dysfunction, micro and macrothrombosis

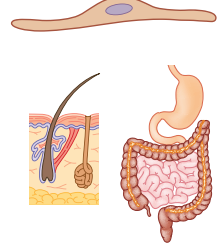

Systemic inflammation and hyperinflammation Gastrointestinal and skin disease

FIGURE 1: Common features in the pathogenesis and clinical manifestations of FD and COVID-19. Attribution of parts of the image: Wolfdog406 at English Wikipedia; Author: Peter van Driel, NL.

Source: https://thenounproject.com/search/? q=kidney\&i=524431

CoV-2 may be heralded by increased pain. Three patients with uncomplicated FD did report pain crisis with fever, complicating otherwise mild COVID-19 disease. Patients with FD may also experience gastrointestinal complications including diarrhea, nausea, vomiting and abdominal pain [26, 28]. Four patients with uncomplicated FD did report increased abdominal pain and/or diarrhea as symptoms associated with COVID-19. As a subset of COVID-19, patients develop gastrointestinal symptoms in addition to the typical respiratory illness; this may also exacerbate underlying FD gastrointestinal symptoms that may require management in a medical setting to avoid dehydration and severe ongoing pain $[29,30]$. One uncomplicated FD patient was hospitalized for observation and received intravenous (IV) fluids to treat dehydration and pain crisis (Table 3 ).

Advanced FD. FD exhibits a high phenotypic variability with a wide clinical spectrum influenced by the nature of the GLA variant and the level of residual $\alpha$-galactosidase among other factors [29-31]. Although as a multisystemic disease, FD typically attacks key organs in tandem resulting in a complex management challenge, this article handles each organ system individually, recognizing that the risks will be cumulative. Patients with advanced FD should be considered a high-risk population, regardless of age. The most severely affected patients do have multiple known risk factors related to renal, cardiac and/or pulmonary disease, immunosuppression following transplant and stroke, which have been identified risk factors of poor outcome in case of COVID-19 [32].

Cardiac disease. Patients with FD may be at an increased risk of cardiovascular morbidity and mortality during SARS-CoV-2 infection, especially in advanced Fabry characterized by cardiomyopathy and malignant arrhythmia [33-37]. In COVID-19, myocardial damage, including fulminant myocarditis with circulatory failure, is a cause of death [38]. Cardiac disease in FD is associated with vascular dysfunction, oxidative stress, cytoskeletal remodeling, inflammation and fibrosis [24, 39, 40, 41] This pre-existent cardiovascular injury may predispose patients 
Table 2. Fabry-specific risk table for severe or critical COVID-19 with recommendations

\begin{tabular}{|c|c|c|c|c|c|}
\hline Condition & $\begin{array}{l}\text { Risk compared } \\
\text { with } \\
\text { general healthy } \\
\text { population }\end{array}$ & $\begin{array}{l}\text { Level of } \\
\text { precaution }\end{array}$ & Preventive measures & $\begin{array}{l}\text { Continue } \\
\text { Fabry- } \\
\text { specific } \\
\text { therapy? }\end{array}$ & $\begin{array}{c}\text { COVID-19-specific therapies } \\
\text { to avoid }\end{array}$ \\
\hline Uncomplicated FD ${ }^{\mathrm{a}}$ & $\begin{array}{l}\text { Potentially higher, } \\
\text { especially } \\
\text { in older males }\end{array}$ & Moderate & $\begin{array}{l}\text { Social distancing, hand hygiene } \\
\text { and outside surgical masks as } \\
\text { for general population may } \\
\text { err on prudent side and do as } \\
\text { for patient with heart and } \\
\text { kidney disease }\end{array}$ & Yes & Hydroxychloroquine/chloroquine \\
\hline $\begin{array}{l}\text { Advanced FD with } \\
\text { kidney disease, } \\
\text { heart disease, } \\
\text { central nervous } \\
\text { system complications }^{\text {and/or lung disease }}{ }^{\text {b }}\end{array}$ & Higher & High & $\begin{array}{l}\text { Enhanced social distancing, } \\
\text { hand hygiene and surgical } \\
\text { or ideally FPP2/N95 masks at } \\
\text { home (if not living alone) } \\
\text { and outside }\end{array}$ & Yes & $\begin{array}{l}\text { Remdesivir in patients with eGFR } \\
<30 \mathrm{~mL} / \mathrm{min} / 1.73 \mathrm{~m}^{2} \\
\text { Hydroxychloroquine/chloroquine } \\
\text { and azithromycin }\end{array}$ \\
\hline Transplantation $^{c}$ & Highest & Highest & $\begin{array}{l}\text { Shielding at home using FPP2/N95 } \\
\text { face masks if not living alone; } \\
\text { hand higiene }\end{array}$ & Yes & $\begin{array}{l}\text { Remdesivir in patients with eGFR } \\
<30 \mathrm{~mL} / \mathrm{min} / 1.73 \mathrm{~m}^{2} \\
\text { Hydroxychloroquine/chloroquine } \\
\text { and azithromycin }\end{array}$ \\
\hline
\end{tabular}

${ }^{a}$ FD with no existing end organ damage (kidney, heart, lung, central nervous system).

${ }^{\mathrm{b}} \mathrm{CKD}$ (pathological albuminuria $>30 \mathrm{mg} / \mathrm{g}$ urinary creatinine) or eGFR $<60 \mathrm{~mL} / \mathrm{min} / 1.73 \mathrm{~m}^{2}$ ) or arrhythmia or severe left ventricular hypertrophy/cardiomegaly or stroke or decreased FEV1.

${ }^{\mathrm{C}}$ Assumed to be on immune suppressants.

RAS either ACEis or ARBs. eGFR, estimated glomerular filtration rate.

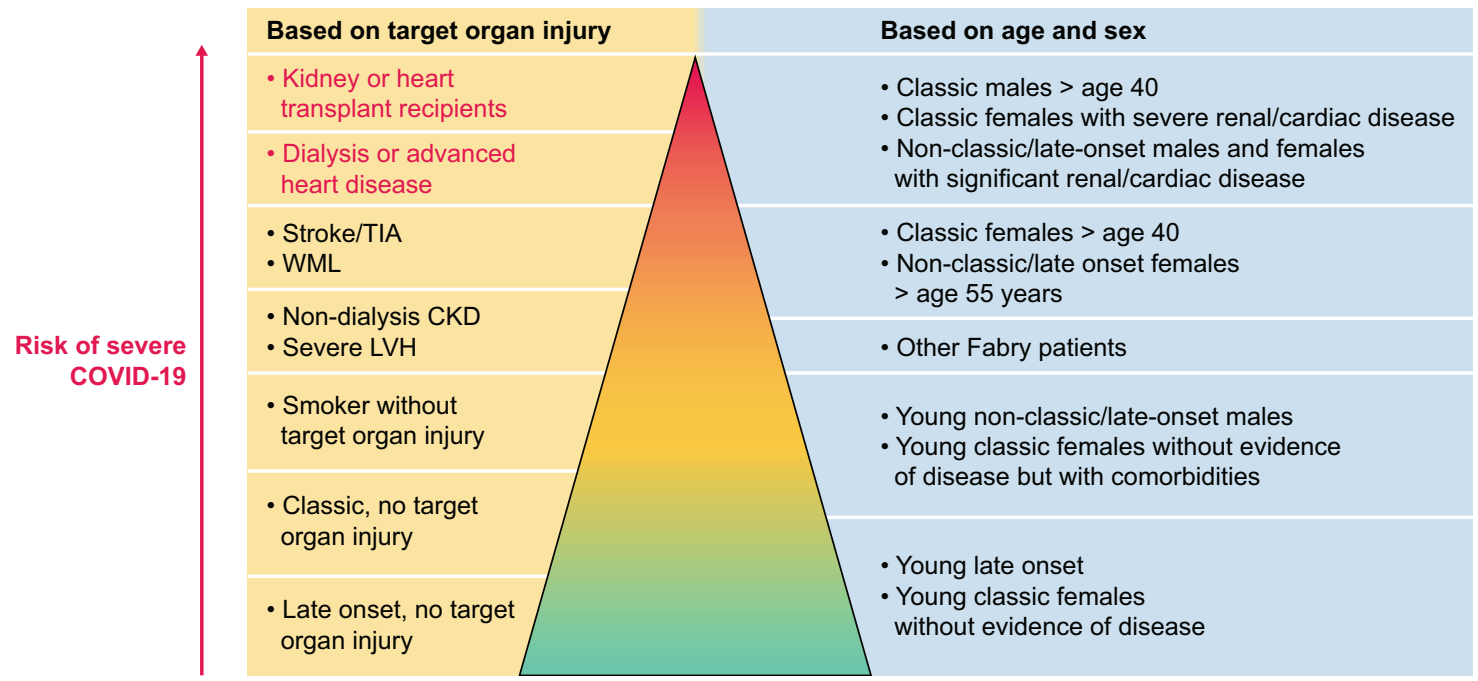

FIGURE 2: Risk of severe or critical COVID-19 in patients with FD. Given the scarcity of Fabry-specific information, this figure has extrapolated knowledge from the general population and the natural history of FD [1, 2]. It shows two scales, depending on whether data are available for specific patients on the extent of target organ injury. When this is known, the 'Based on target organ injury' scale should be used. When this is unknown, the 'Based on age and sex' scale provides some guidance. TIA, transient ischaemic attack; WML, white matter lesion; LVH, left ventricular hypertrophy.

with FD to more severe cardiovascular COVID-19 manifestations as seen in Patients \#5, \#8 and \#15 in Table 3.

Chronic kidney disease. FD causes a proteinuric nephropathy that may need renal replacement therapy (RRT; dialysis or transplantation) at a mean age of 40 years [42, 43]. Individuals with CKD, particularly those on RRT, are at higher risk of severe or critical COVID-19 and death $[8,44]$. Additionally, they are at higher risk of acute-on-chronic renal injury, a frequent occurrence in patients with COVID-19, particularly at the time of intubation, with the need for dialysis, a major poor prognostic factor.
A further potential issue is that COVID-19 may cause special forms of focal and segmental glomerulosclerosis (FSGS), especially in African-Americans carrying specific APOL1 alleles [45]. Thus, COVID-19 may potentially aggravate podocyte injury and/ or accelerate the FSGS observed in FD. Patients with FD and COVID-19 should be monitored for this possibility.

Neurologic complications. Cerebrovascular complications in FD include acute ischemia (small and large vessel stroke), hemorrhage and chronic cerebrovascular disease [46]. Ischemic stroke has been reported as a frequent complication in COVID-19 


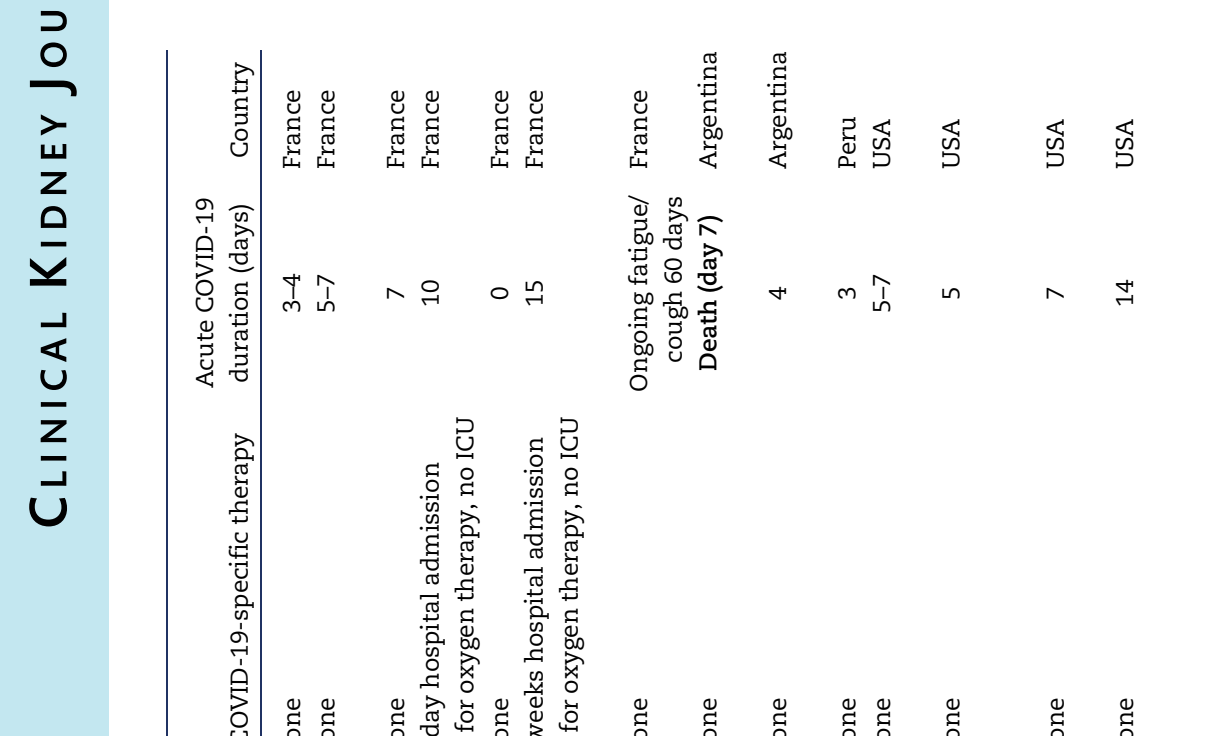
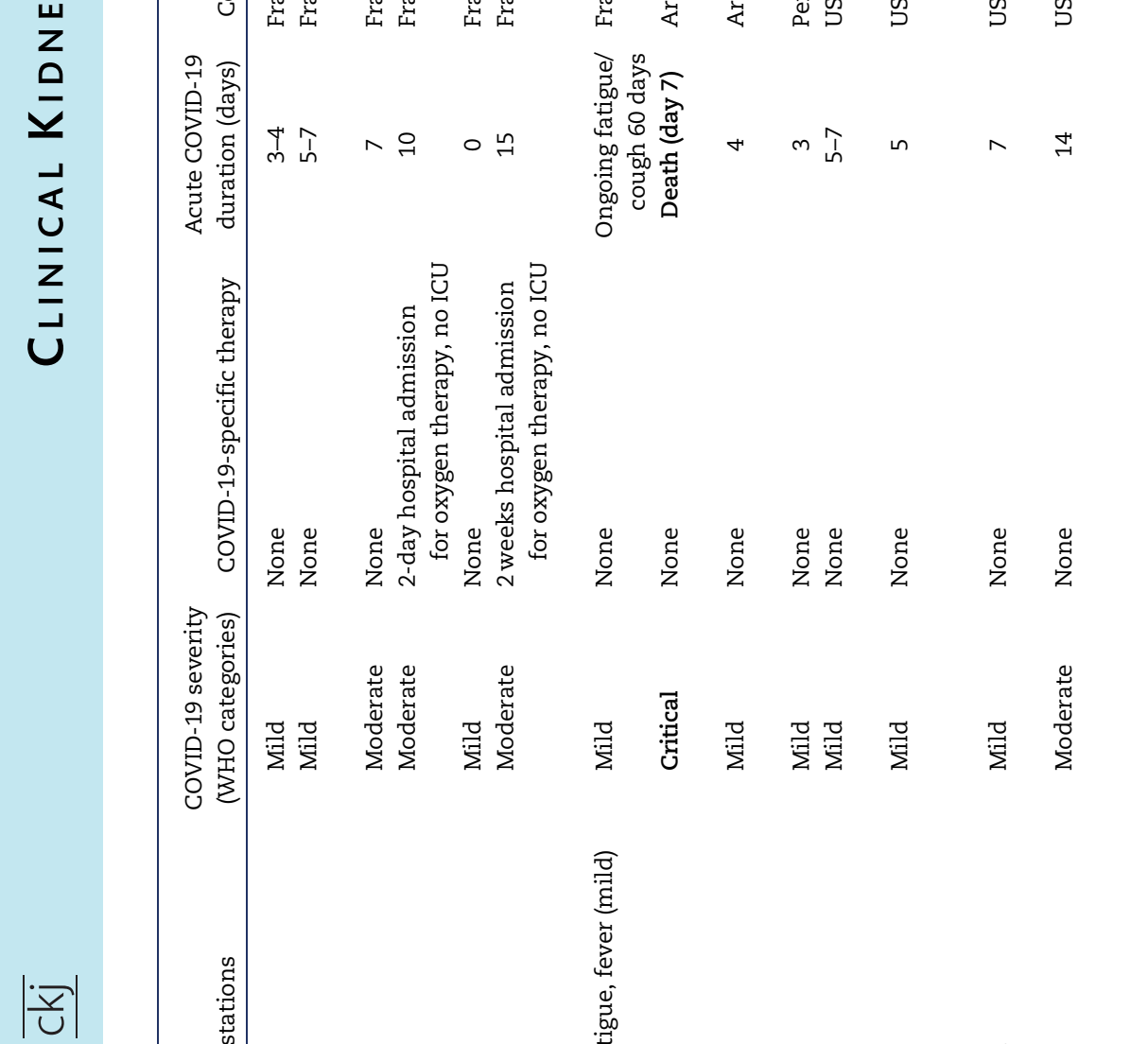

密矛

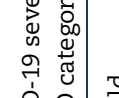

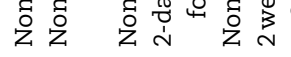

它虽

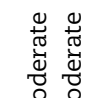

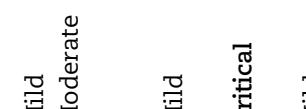

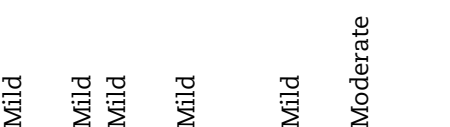

凹

峁

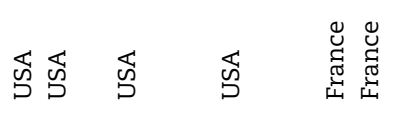
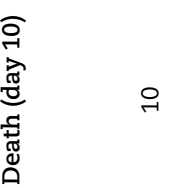

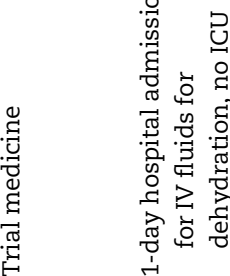

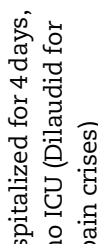

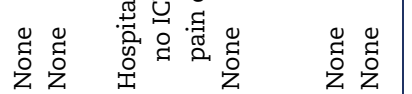

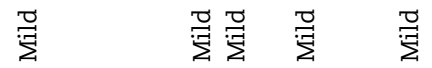

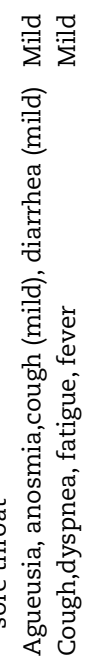

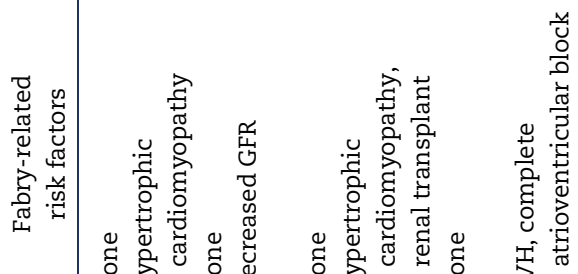

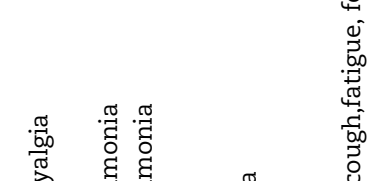
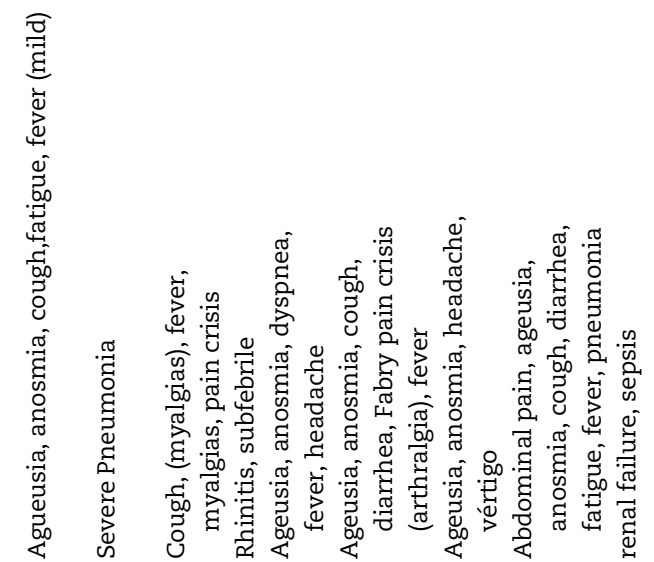

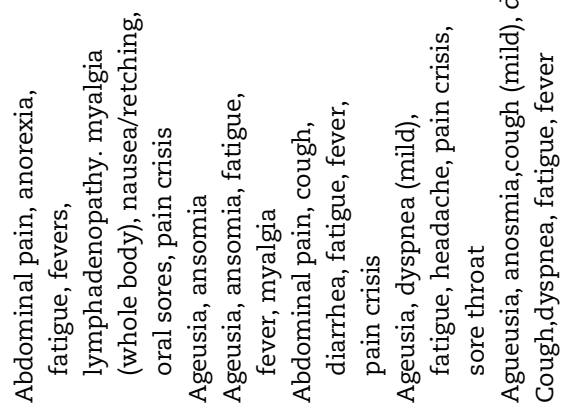

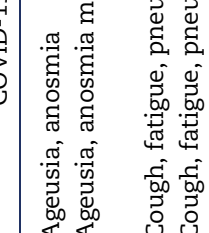

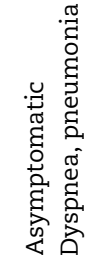

zิ 完号

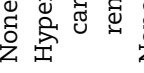

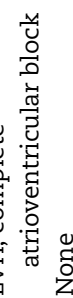

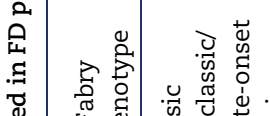
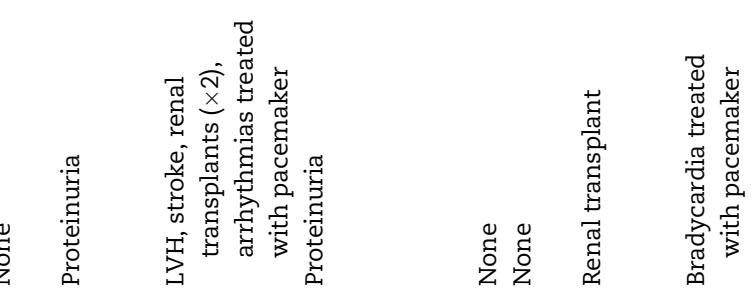

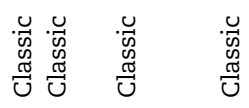

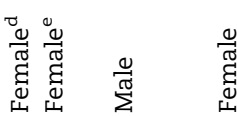

焉 
patients [47]. Due to possible common pathogenetic mechanisms, clinicians should be aware that patients with FD may be potentially at a major risk for stroke manifestation as a COVID19 complication [48]. Stroke in patients with FD and COVID-19 should be treated with acute therapy and secondary prevention therapy $[48,49]$. Furthermore, more recent data indicate that neurological manifestations in patients with COVID-19 may have a broader spectrum, including PNS involvement (there are emerging reports of Guillain-Barré syndrome) [47].

Neuropsychiatric manifestations. Depression develops in up to $46 \%$ of patients with FD and is frequently associated with pain [4951]. Depression, anxiety and post-traumatic stress disorder can be also be found with increased frequency in patients with COVID-19 and individuals in the general population in response to enforced lockdown and stress due to the pandemic [52-54]. Clinicians taking care of patients with FD and COVID-19 should monitor carefully and manage mood changes of their patients, since long-term post-traumatic stress disorder has been recognized in Middle East respiratory syndrome coronavirus survivors in Korea [55]. Anxiety, depression or fear of entering an infusion center also can lead to an arbitrary cessation of ERT in patients with FD.

Transplant. Patients with FD with advanced disease may have received a kidney or heart transplant. Organ transplant recipients on immune suppressants should be considered at an increased risk of severe or critical COVID-19 and early reports support this heightened risk, with mortality rates in immunosuppressed patients around $25-30 \%$ [56]. The risk is likely higher in the early post-transplant period when immune suppressant dose is higher. Many centers have temporarily discontinued transplant programs in the more active phase of the epidemic and slowly resumed them once medical supplies and care circuits could be re-established safety [56]. All transplant patients with COVID-19 should be admitted to hospital as they may present with milder symptoms that progress rapidly resulting in increased risk for unanticipated death at home [56]. However, emphasis should be placed on prevention. Routine shielding and use of N95/FPP2 face masks are recommended on top of other preventive measures (Figure 1).

Pulmonary disease. Patients with FD may have silent or overt pulmonary involvement, putting them potentially at increased risk of severe or critical COVID-19 pulmonary manifestations, the most common cause of death in COVID-19 patients. Symptoms and signs of pulmonary involvement include dyspnea, wheezing and dry cough [57]. The proposed mechanisms for the development of airway obstruction in FD include loss of lung elastic recoil, and airway inflammation and narrowing related to accumulation of glycosphingolipid and hyperplasia in airway epithelial and smooth muscle cells [57]. Thus, target cells partially overlap with those in COVID-19. Obstructive lung disease was present in $46 \%$ of patients with FD, this being up to 10 -fold more frequent than in the background population $[58,59]$.

\section{MEDICATIONS COMMONLY USED IN THE TREATMENT OF FD AND INTERACTION WITH COVID-19}

The interaction of COVID-19 with specific therapies for FD has not been formally studied in patients with FD; however, mechanism of action of these medications and any available data is discussed in this section.

\section{Renin-angiotensin system blockade}

Many patients with FD are taking renin-angiotensin system (RAS) blockers as antiproteinuric, nephroprotective and cardioprotective agents [26]. Early in the course of the pandemic, there was controversy on the benefit-risk balance of RAS blockade. Since SARS-CoV-2 enters host cells by binding to ACE2 at the cell membrane, and experimental animal data suggest that RAS blockade could increase ACE2 expression in cardiovascular and renal systems, it was hypothesized that treatment with angiotensin II receptor blockers (ARB) and angiotensin-converting enzyme inhibitors (ACEIs) might be deleterious by facilitating viral entry into cells [60]. This hypothesis led patients and physicians to stop ARBs/ACEIs, overlooking the alternative hypothesis that, by increasing soluble ACE2 (sACE2), RAS blockade might be protective by providing a decoy receptor for SARS-CoV-2 (Supplementary data, Figure S1A). In clinical trials exploring recombinant human SACE2 to treat COVID-19, no evidence has been found so far of either an adverse or beneficial effect of RAS blockade on COVID-19, and scientific societies have emphasized the risks of stopping RAS blockade [61-63]. A single manuscript claiming a survival benefit for RAS blockade in COVID-19 has been retracted [64].

\section{ERT}

Whether COVID-19 has an impact on the effectiveness of ERT for FD is unknown, but some characteristics of SARS-CoV-2 infection should be discussed. Agalsidase is internalized by endocytosis from the extracellular space into endosomes through cell surface receptors for mannose-6-phosphate (M6PR) as well as through megalin or sortilin. The acidic $\mathrm{pH}$ of late endosomes induces dissociation of the agalsidase-M6PR complexes, and agalsidase is delivered to lysosomes, while the receptor is recycled to the cell surface $[65,66]$. A massive occupation of endosomes by competitors or binding of other molecules to their cognate receptors may interfere with the endocytic process or downregulate receptors through their internalization into lysosomes. Thus, both the SARS-CoV-2 and agalsidase share a step of membrane fusion for cellular entrance and there is potential competition between SARS-CoV-2 and ERT [67].

\section{Chaperone therapy}

Whether COVID-19 has an impact on the effectiveness of chaperone for FD is unknown. Much like ERT, there is also possible competition between SARS-CoV-2 and $\alpha$-galactosidase A enzyme coupled with the pharmacological chaperone [67].

\section{COVID-19 THERAPY AND FD}

Current therapy of COVID-19 is for the most part empirical and based on treatment of disease symptoms [68]. Most medications and therapies used to treat COVID, except for chloroquine and hydroxychloroquine, do not have contraindications in patients with FD; however, each medication should undergo a safety profile review prior to initiating therapy in a patient with FD [69]. 


\section{Antiviral therapy}

This is aimed at interfering with viral cell entry into cells or viral replication. Remdesivir has been found to shorten the time to recovery in adults hospitalized with COVID-19 and evidence of lower respiratory tract infection [70]. Additional antivirals or drugs that potentially interfere with viral replication are or have been commonly used include antivirals (e.g. lopinavir/ritonavir). Although not specifically studied in FD, remdesivir is potentially nephrotoxic and is not recommended in individuals with glomerular filtration rate $<30 \mathrm{~mL} / \mathrm{min} / 1.73 \mathrm{~m}^{2}$ [71]. Otherwise, there is no contraindication for remdesivir in FD [69].

Although no longer a preferred treatment option for COVID19 , the early danger recognized in patients with FD using chloroquine/hydroxychloroquine with its risks to inactivate Fabryspecific therapies (ERT and chaperone therapy), prolong QT intervals and trigger torsade de pointes underlines the importance of completing a safety assessment of investigational treatment options [72-77].

\section{Anti-inflammatory drugs}

These medications are aimed at decreasing the adverse impact of the cytokine storm. Drugs used so far include steroids, antiinterleukin 6 (anti-IL-6) drugs (mainly tocilizumab) and janus kinase inhibitors (such as baricitinib), which, together with other approaches, are undergoing clinical trials [69]. Cytokines are usually elevated in patients with FD at low but steady levels, but how that impacts the risk of a cytokine storm during a COVID-19 infection is unknown [78]. Tocilizumab and related anti-IL-6 drugs have not been studied specifically in FD.

\section{Anti-thrombotic strategies}

This consists mainly of low molecular weight heparin for COVID-19 patients including those not requiring admission. There is no FD-specific contraindication for heparin; however, many patients with FD may already be on aspirin, coumadin or other anti-thrombotic medications.

\section{Organ replacement/support}

The need for oxygen therapy is a common indication for hospital admission. This treatment was used successfully in two patients with FD in our data series. Beyond such supplementation, critical COVID-19 cases may need full cardiovascular, ventilatory and kidney support or replacement, including vasoactive drugs, ventilators or extracorporeal membrane oxygenation and RRT. Individuals with FD can be treated as their disease state indicates with regards to oxygen, transplant and life maintaining support. Living donors should be tested for FD prior to transplant consideration [26,69]. In several cities, hospitals were overwhelmed by COVID-19 patients and access to intensive care, ventilators or dialysis could not be guaranteed for all those who needed it. This group strongly recommends that diagnosis of FD should not be the basis for denying intensive care, organ replacement or supportive therapy when indicated. Peritoneal dialysis is an acceptable alternative when hemodialysis or hemofiltration cannot be offered [79].

\section{Convalescent plasma}

COVID-19 convalescent plasma has been used to treat the disease [80]. One patient with critical COVID-19 and FD did have an initial positive response to treatment early in disease course, but it did not have a longer term positive impact on COVID-19 progression. There is no FD-specific contraindication for use of convalescent plasma in patients with FD.

\section{Exogenous pulmonary surfactant}

The pathophysiological similarities between the infant respiratory distress syndrome and the lung involvement in COVID-19 have led clinicians to hypothesize that the administration of pulmonary surfactant might be of help in patients with COVID19 requiring ventilator support; a clinical trial of nebulized surfactant therapy has been recently launched [81]. There is no FDspecific contraindication for use of pulmonary surfactant in patients with FD.

\section{MITIGATING THE RISK OF SARS-COV-2 INFECTION IN FD WHILE CONTINUING ACCESS TO SPECIFIC THERAPY}

Clinical monitoring and enzyme replacement or chaperone therapy are needed for optimal outcomes in FD [82]. In this regard, the risk of developing COVID-19 should be weighed against the risks of temporary interruption of ERT. Since the primary mode of SARS-CoV-2 transmission is from person-to-person (through respiratory droplets or direct and indirect contacts), patients with FD should be advised to take preventive measures [83-85]. Additionally, healthcare institutions should also take measures to provide healthcare safely. It is the authors' opinion that for most patients with FD, the benefits of continuing ERT without interruption outweigh the risk of contracting SARS-CoV-2 at home or in a secure healthcare setting [86].

\section{Decreasing healthcare-associated risks}

Healthcare facilities are enriched for both people more likely to be currently infected and for individuals at higher risk for serious complications. It is likely that spread to employees in these settings is increased [87]. While harder to study and demonstrate, it is also likely that spread between patients is increased, particularly in waiting rooms or other shared spaces. This has led some patients with FD to conclude that the risk of exposure to COVID-19 is high enough to consider interruption of treatment and cancellation of evaluations. However, there are ways to minimize this risk and these have been summarized in International Organization for Standardization standards on the topic [88].

- Telemedicine. During closures of physical medical centers for in-person appointments and assessments at peak infection times, telemedicine appointments allow consultation with key healthcare providers who can make the decision to delay or continue specific assessments and treatments on an individual basis.

- Home infusions are part of efforts to limit the need of patients with FD to leave home without compromising healthcare at the peak of the pandemic. They may decrease the risk associated with transportation to and from infusion centers. For this, the person administering the infusions should strictly follow the recommended hygiene measures and use appropriate personal protective equipment (PPE), including mask, gloves and gowns, to minimize risk.

- Establishment of safe in-center infusion protocols. Risks can be decreased in center-based care by use of masks, screening employees and patients for fever and other COVID-19related symptoms, minimizing the number of people 
accompanying patients, rapid rooming, adequate airing and use of appropriate hand washing and PPE [89]. The WHO has published technical guidelines on infection prevention and control and most institutions and out-patient centers have instituted very strict infection control protocols resulting in lower transmission rates [4]. A mixture of these measures has been used in real-world experiences for FD therapy (Supplementary data, Table S2).

Based on the authors' clinical experience, patients with FD do not seem to be at higher risk of becoming infected with the SARSCoV-2 than their household contacts and relatives without FD.

\section{GENERAL APPROACH TO PREVENTION OF COVID-19}

Until a vaccine or a safe pre-exposure prophylaxis protocol are developed, prevention of COVID-19 depends on social distancing, the use of face masks, hand washing and respiratory hygiene.

Measures to prevent SARS-CoV-2 infection involve several interrelated layers. Those involving actions by patients and their families and those involving government intervention are summarized in Supplementary data, Table S2. The main routes of acquiring the virus in the community are via respiratory droplets in the air or present on surfaces $[85,90]$. It is now acknowledged that the SARS-CoV-2 RNA can be detected in the upper respiratory tract up to 5 days before symptoms develop and asymptomatic individuals may transmit the disease [91]. Under pandemic conditions, everybody, including healthcare personnel, should be considered potentially infectious and take precautions.

\section{Specific considerations for prevention of intrafamilial} transmission

Since FD is a genetic disease, multiple family members with FD may be present in a single household [92]. Face mask use by the primary case and family contacts before the primary case developed symptoms was $80 \%$ effective in reducing transmission, an impact similar to daily use of chlorine or ethanol-based disinfectant in households [93]. However, wearing a mask after illness onset in the primary case was not significantly protective. Although PCR positivity does not mean patients are infectious, an additional consideration is that individuals with decreased kidney function may remain viremic for longer than other patients, with up to $90 \%$ of dialysis patients still PCR-positive a median of 19 days post-diagnosis [94]. Patient \#16 in our series was PCR-positive 30 days post-diagnosis (Table 3). The recommended time before contact with other FD family members should be reviewed with a clinician prior to resumption.

\section{CALL FOR AN INTERNATIONAL REGISTRY FOR COVID-19 IN PATIENTS WITH FD}

Management recommendations in this article are based on FD experts' experience with COVID-19 and individual case outcomes. However, the rapidly evolving understanding of the impact and treatment of COVID-19 requires clinicians caring for patients with FD to adjust their patient care plans. Whether patients with FD have an increased risk of SARS-CoV-2 infection or of severe/critical COVID-19 remains an unsolved question. Thus, it is important to systematically collect data regarding exposures and confirmed infection to better inform optimal management and assess potential risks to this population. To compile as much data as possible to inform best practices, an international registry for patients with FD infected by SARSCoV-2 should be established (Box 2). Such a registry would allow scientists and clinicians to explore the impact of genetic variations that have been associated with more severe COVID-19 in the general population [95-98]. It is also important to learn of any repeat infections or COVID-19 antibody titers. Analysis of this registry data will pave the way to providing information based on real-time updated data about the impact of COVID-19 on patients living with FD and how to manage infected patients across the disease spectrum.

\section{CONCLUSIONS}

Our limited data are insufficient to confirm or reject the hypothesis that all patients with FD may be at increased risk of severe or critical COVID-19. However, it appears that patients with advanced FD characterized by a kidney or cardiac transplant, use of immunosuppressive medications and/or severe cardiovascular complications may be at risk of severe or critical COVID-19 and poor outcome, as is the case for individuals in the general population. Given these theoretical considerations and the spectrum of individual susceptibility to infection, it is wise that patients with FD in the higher risk groups strictly comply with the SARS$\mathrm{CoV}-2$ general safety precautions. Continued specific therapy for FD during the pandemic is endorsed, even if it requires visits to a medical center every 2 weeks for ERT administration, since its benefits outweigh the perceived risk of developing COVID-19 in secure healthcare settings. More data are needed to understand infectivity, the manifestations and the natural history of SARSCoV-2 infection in patients with FD, but while evidence-based recommendations are not available the need for strict adherence to the protective guidelines issued by the WHO should be emphasized, especially hand hygiene, social distancing, airing and face mask use [4]. Until a vaccine becomes available, keep on guard for COVID-19 for the foreseeable future. Physicians caring for patients with FD should be aware of the evolving local epidemiological conditions and be especially careful in the autumn and winter, when increased indoor activities and family gatherings may favor new waves of SARS-CoV-2 epidemic.

Box 1 Key messages for FD in the context of the SARS-CoV-2 pandemic

- Currently, prevention of infection is the main current weapon against SARS-CoV-2.

- The pathogenesis and target organs for COVID-19 shares some features with FD.

- Pre-existent tissue injury may predispose patients with advanced FD to a more severe course of COVID-19.

- Patients with FD should continue to receive full therapy for FD, including ERT, chaperone therapy and/or reninangiotensin blockers.

- A diagnosis of FD should not preclude providing full therapeutic and organ support as needed for patients with severe or critical COVID-19.

- Some drugs used to treat COVID-19 may have a negative safety profile for patients with prior heart conditions and Fabry physicians should be consulted prior to prescription. 
Box 2 Key data fields for a registry of SARS-CoV-2 infection in patients with FD

- Demographic information

- Genotype (pathogenic variant in the GLA gene)

- Primary therapy as appropriate and date initiated

- Date and type of positive COVID-19 testing

- Pre-COVID-19 stage of renal disease

- Pre-COVID-19 cardiac, pulmonary and cerebrovascular status

- Comorbidities

- Pre-COVID-19 use of angiotensin-converting enzyme inhibitors

- Initial COVID-19 symptoms

- COVID-19 treatments

- COVID-19 complications

- Outcome

\section{SUPPLEMENTARY DATA}

Supplementary data are available at ckj online.

\section{ACKNOWLEDGEMENTS}

The authors would like to recognize our patients living with FD, who challenge us daily to better understand the impact of the disease on their lives.

\section{FUNDING}

A.O. research is supported by FIS/FEDER funds [PI19/00815, DTS18/00032, ERA-PerMed-JTC2018 (KIDNEY ATTACK AC18/ 00064 and PERSTIGAN AC18/00071), ISCIII-RETIC REDinREN RD016/0009], Sociedad Española de Nefrología, FRIAT, Comunidad de Madrid en Biomedicina B2017/BMD-3686 CIFRA2-CM. D.P.G. is supported by the French 'Plan National Maladies Rares'.

\section{AUTHORS' CONTRIBUTIONS}

All persons who meet authorship criteria are listed as authors, and all authors certify that they have participated sufficiently in the work to take public responsibility for the content, including participation in the concept, design, research, writing or revision of the manuscript. Conception and design of manuscript were done by D.A.L., D.P.G., R.J.H., A.O., J.P.O. and J.M.P. Research, provision of data, drafting and revision of manuscript were carried out by A.P.B., G.H.C., G-R..H., D.A.L., D.P.G., R.J.H., D.-M.N., A.O., J.P.O., J.M.P., H.T., M.T. and W.R.W. All authors approved the version of the manuscript to be published.

\section{CONFLICT OF INTEREST STATEMENT}

A.P.B. has received research support from Sanofi-Genzyme, consulting fees and lecture fees from Amicus Therapeutics, Freeline Therapeutics and Takeda-Shire. D.P.G. has received speaker's and consulting fees and research support from Amicus Therapeutics, Sanofi-Genzyme and Takeda-Shire.
G.H.C. has received lecture and travel honoraria from Sanofi-Genzyme, Pint Pharma and Takeda-Shire. G.-R.H. has no conflicts of interest to disclose. R.J.H. consults with Amicus Therapeutics, AVROBIO, Chiesi, Protalix BioTherapeutics, Sangamo, Sanofi-Genzyme and TakedaShire, and has been an investigator in clinical trials sponsored by Amicus Therapeutics, Protalix BioTherapeutics, Sanofi-Genzyme and Takeda-Shire. He is on the speakers bureau for Alexion, Amicus Therapeutics, Sanofi-Genzyme. These activities have been monitored and found to be in compliance with the conflict of interest policies at Cincinnati Children's Hospital Medical Center. D.A.L. is chair of the North American Fabry Registry Board, consults with Sanofi-Genzyme, and has been an investigator and coordinator in clinical trials sponsored by 4DMT, Amicus Therapeutics, Freeline, Protalix BioTherapeutics, Sangamo, Sanofi-Genzyme and Takeda-Shire. These activities have been monitored and found to be in compliance with the conflict of interest policies at the Emory University School of Medicine. D.-M.N. has no conflicts of interest to disclose. A.O. has received consulting fees, and research support from Sanofi-Genzyme, consulting fees from Freeline and lecture fees and/or travel support from Sanofi-Genzyme, Amicus Therapeutics and Takeda-Shire. J.P.O. is the member of the European Advisory Board of the Fabry Registry and has received honoraria for consultancies, honoraria for disease registry advisory board, honoraria for lecturing and support for research from Sanofi-Genzyme. He also has received consulting and/or lecturing honoraria from Protalix/ Chiesi and Takeda-Shire. He has received support for travel and accommodation from Amicus Therapeutics, SanofiGenzyme and Takeda-Shire. J.M.P. has received honoraria for presentations and board meetings from Amicus Therapeutics, Sanofi-Genzyme and Takeda-Shire. H.T. has received honoraria for lectures on FD from Sanofi-Genzyme. M.T. has received lecture honoraria, conference support, advisory board fees and research support from SanofiGenzyme, Takeda-Shire, Amicus Therapeutics, Idorsia and AVROBIO. W.R.W. consults for Sanofi-Genzyme and TakedaShire and is an investigator in clinical trials for FD sponsored by 4DMT, Amicus Therapeutics, BioMarin, Freeline, Idorsia, Protalix BioTherapeutics, Sangamo Therapeutics and Sanofi-Genzyme. These activities are monitored and are in compliance with the conflict of interest policies at the Emory University School of Medicine.

\section{DATA AVAILIBILITY STATEMENT}

The data underlying this article are available in the article and in its online supplementary material.

\section{REFERENCES}

1. Germain DP. Fabry disease. Orphanet J Rare Dis 2010; 5: 30

2. Arends M, Wanner C, Hughes D et al. Characterization of classical and nonclassical Fabry disease: a multicenter study. J Am Soc Nephrol 2017; 28: 1631-1641

3. Germain DP, Oliveira JP, Bichet DG et al. Use of a rare disease registry for establishing phenotypic classification of previously unassigned GLA variants: a consensus classification system by a multispecialty Fabry disease genotype-phenotype workgroup. J Med Genet 2020; 57: 542 
4. World Health Organization. Clinical Management of COVID-19: interim Guidance, 27 May 2020. https://www.who.int/publica tions/i/item/clinical-management-of-covid-19 (16 October 2020, date last accessed)

5. Russell TW, Hellewell J, Jarvis CI et al. Estimating the infection and case fatality ratio for coronavirus disease (COVID19) using age-adjusted data from the outbreak on the Diamond Princess cruise ship, February 2020. Euro Surveill 2020; 25: doi: 10.2807\%2F1560-7917.ES.2020. 25.12.2000256 (13 November 2020, date last accessed)

6. Oran DP, Topol EJ. Prevalence of asymptomatic SARS-CoV-2 infection: a narrative review. Ann Intern Med 2020; 173: 362-367

7. Yang J, Zheng Y, Gou X et al. Prevalence of comorbidities in the novel Wuhan coronavirus (COVID-19) infection: a systematic review and meta-analysis. Int J Infect Dis 2020; 94: 91-95

8. Centers for Disease Control and Prevention (CDC) COVID-19 Response Team. Preliminary estimates of the prevalence of selected underlying health conditions among patients with Coronavirus Disease 2019 - United States, February 12March 28, 2020. MMWR Morb Mortal Wkly Rep 2020; 69: 382-386

9. Williamson EJ, Walker AJ, Bhaskaran $\mathrm{K}$ et al. Factors associated with COVID-19-related death using OpenSAFELY. Nature 2020; 584: 430-436

10. Livingston E, Bucher K. Coronavirus Disease 2019 (COVID-19) in Italy. JAMA 2020; 323: 1335

11. Wu Z, McGoogan JM. Characteristics of and important lessons from the coronavirus disease 2019 (COVID-19) outbreak in China: summary of a report of 72314 cases from the Chinese Center for Disease Control and Prevention. JAMA 2020; 323: 1239

12. Coronaviridae Study Group of the International Committee on Taxonomy of Viruses. The species severe acute respiratory syndrome-related coronavirus: classifying 2019$\mathrm{nCoV}$ and naming it SARS-CoV-2. Nat Microbiol 2020; 5: 536-544

13. Zhu N, Zhang D, Wang $\mathrm{W}$ et al. A novel coronavirus from patients with pneumonia in China, 2019. N Engl J Med 2020; 382: 727-733

14. Lu R, Zhao X, Li J et al. Genomic characterisation and epidemiology of 2019 novel coronavirus: implications for virus origins and receptor binding. Lancet 2020; 395: 565-574

15. Batlle D, Soler MJ, Sparks MA et al. Acute kidney injury in COVID-19: emerging evidence of a distinct pathophysiology. J Am Soc Nephrol 2020; 31: 1380-1383

16. Danilczyk U, Penninger JM. Angiotensin-converting enzyme II in the heart and the kidney. Circ Res 2006; 98: 463-471

17. Lamers MM, Beumer J, van der Vaart J et al. SARS-CoV-2 productively infects human gut enterocytes [published online ahead of print May 1, 2020]. Science 2020; 369: 50-54

18. Qi F, Qian S, Zhang $S$ et al. Single cell RNA sequencing of 13 human tissues identify cell types and receptors of human coronaviruses. Biochem Biophys Res Commun 2020; 526: $135-140$

19. Zhang H, Penninger JM, Li Y et al. Angiotensin-converting enzyme 2 (ACE2) as a SARS-CoV-2 receptor: molecular mechanisms and potential therapeutic target. Intensive Care Med 2020; 46: 586-590

20. Demuth K, Germain DP. Endothelial markers and homocysteine in patients with classic Fabry disease. Acta Paediatr Suppl 2007; 91: 57-61
21. Ackerman M, Verleden SE, Kuehnel M et al. Pulmonary vascular endothelialitis, thrombosis, and angiogenesis in Covid-19. N Engl J Med 2020; 383: 120-128

22. Varga Z, Flammer AJ, Steiger $P$ et al. Endothelial cell infection and endothelitis in COVID-19. Lancet 2020; 395: 1417-1418

23. Riccio E, Pieroni M, Limoneglli G et al. Impact of COVID-19 pandemic on patients with Fabry disease: an Italian experience. Mol Genet Metab 2020 https://doi.org/10.1016/j.ymgme. 2020.07.008 (13 November 2020, date last accessed)

24. Yogasundaram H, Nikhanj A, Putko BN et al. Elevated inflammatory plasma biomarkers in patients with Fabry disease: a critical link to heart failure with preserved ejection fraction. J Am Heart Assoc 2018; 7: e009098

25. Kissling S, Rotman S, Gerber C et al. Collapsing glomerulopathy in a COVID-19 patient. Kidney Int 2020 (published online ahead of print, 15 April 2020; doi: 10.1016/j.kint.2020.04.006)

26. Ortiz A, Germain DP, Desnick RJ et al. Fabry disease revisited: management and treatment recommendations for adult patients. Mol Genet Metab 2018; 123: 416-427

27. Politei JM, Bouhassira D, Germain DP et al. Pain in Fabry disease: practical recommendations for diagnosis and treatment. CNS Neurosci Ther 2016; 22: 568-576

28. Hopkin RJ, Feldt-Rasmussen U, Germain DP et al. Improvement of gastrointestinal symptoms in a significant proportion of male patients with classic Fabry disease treated with agalsidase beta: a Fabry Registry analysis stratified by phenotype. Mol Genet Metab Report 2020; 25: 10.1016/j.ymgmr.2020.100670 (13 November 2020, date last accessed)

29. Xiao F, Tang M, Zheng X et al. Evidence for gastrointestinal infection of SARS-CoV-2. Gastroenterology 2020; 158: 1831-1833.e3

30. Lin L, Jiang $X$, Zhang $Z$ et al. Gastrointestinal symptoms of 95 cases with SARS-CoV-2 infection. Gut 2020; 69: 997-1001

31. Germain DP, Biasotto M, Tosi $M$ et al. Fluorescence-assisted mismatch analysis (FAMA) for exhaustive screening of the alpha-galactosidase A gene and detection of carriers in Fabry disease. Hum Genet 1996; 98: 719-726

32. Hopkin RJ, Cabrera G, Charrow J et al. Risk factors for severe clinical events in male and female patients with Fabry disease treated with agalsidase beta enzyme replacement therapy: data from the Fabry Registry. Mol Genet Metab 2016; 119: 151-159

33. Linhart A, Kampmann C, Zamorano JL et al.; on behalf of European FOS Investigators. Cardiac manifestations of Anderson-Fabry disease: results from the international Fabry outcome survey. Eur Heart J 2007; 28: 1228-1235

34. Linhart A, Germain DP, Olivotto I et al. An expert consensus document on the management of cardiovascular manifestations of Fabry disease. Eur J Heart Fail 2020; 22: 1076-1096

35. Senechal M, Germain DP. Fabry disease: a functional and anatomical study of cardiac manifestations in 20 hemizygous male patients. Clin Genet 2003; 63: 46-52

36. Shah JS, Hughes DA, Sachdev B et al. Prevalence and clinical significance of cardiac arrhythmia in Anderson-Fabry disease. Am J Cardiol 2005; 96: 842-846

37. Cabrera G. Update on coronary involvement in Fabry disease. JIEMS 2016; 4: 1-5

38. Ruan $\mathrm{Q}$ Yang $\mathrm{K}$, Wang $\mathrm{W}$ et al. Clinical predictors of mortality due to COVID-19 based on an analysis of data of 150 patients from Wuhan, China. Intensive Care Med 2020; 46: 1294-1297. 
39. Hollander Z, Dai DL, Putko BN et al. Gender-specific plasma proteomic biomarkers in patients with Anderson-Fabry disease. Eur J Heart Fail 2015; 17: 291-300

40. Moore DF, Krokhin OV, Beavis RC et al. Proteomics of specific treatment-related alterations in Fabry disease: a strategy to identify biological abnormalities. Proc Natl Acad Sci USA 2007; 104: 2873-2878

41. Bolsover FE, Murphy E, Cipolotti L et al. Cognitive dysfunction and depression in Fabry disease: a systematic review. J Inherit Metab Dis 2014; 37: 177-187

42. Ortiz A, Oliveira JP, Waldek S et al.; Fabry Registry. Nephropathy in males and females with Fabry disease: crosssectional description of patients before treatment with enzyme replacement therapy. Nephrol Dial Transplant 2008; 23: 1600-1607

43. Ortiz A, Cianciaruso B, Cizmarik $M$ et al. End-stage renal disease in patients with Fabry disease: natural history data from the Fabry Registry. Nephrol Dial Transplant 2010; 25: 769-775

44. Rincón A, Moreso F, López-Herradón A et al. The keys to control a COVID-19 outbreak in a hemodialysis unit. Clin Kidney J 2020; 13: 542-549

45. Couturier A, Ferlicot S, Chevalie $\mathrm{K}$ et al. Indirect effects of severe acute respiratory syndrome coronavirus 2 on the kidney in coronavirus disease patients. Clin Kidney J 2020; 13 : 347-353

46. Kolodny E, Fellgiebel A, Hilz MJ et al. Cerebrovascular involvement in Fabry disease: current status of knowledge. Stroke 2015; 46: 302-313

47. Pezzini A, Padovani A. Lifting the mask on neurological manifestations of COVID-19. Nat Rev Neurol 2020; 24: 1-9

48. Reisin RC, Rozenfeld P, Bonardo P. Fabry disease patients have an increased risk of stroke in the COVID-19 ERA. A hypothesis. Med Hypotheses 2020; 144: 110282

49. Mancuso M, Arnold M, Bersano A et al. Monogenic cerebral small-vessel diseases: diagnosis and therapy. Consensus recommendations of the European Academy of Neurology. Eur J Neurol 2020; 27: 909-927

50. Laney DA, Gruskin DJ, Fernhoff PM, Cubells JF et al. Socialadaptive and psychological functioning of patients affected by Fabry disease. J Inherit Metab Dis 2010; 33: 73-81

51. Ali N, Gillespie S, Laney D. Treatment of depression in adults with Fabry disease. JIMD Rep 2018; 38: 13-21

52. Rogers JP, Chesney E, Oliver D. Psychiatric and neuropsychiatric presentations associated with severe coronavirus infections: a systematic review and meta-analysis with comparison to the COVID-19 pandemic. Lancet Psychiatry 2020; 7: 611-627

53. Varatharaj A, Thomas N, Ellul MA et al. CoroNerve Study Group. Neurological and neuropsychiatric complications of COVID-19 in 153 patients: a UK-wide surveillance study. Lancet Psychiatry 2020; 7: 875-882

54. Pierce M, Hope H, Ford T et al. Mental health before and during the COVID-19 pandemic: a longitudinal probability sample survey of the UK population. Lancet Psychiatry 2020; 7: 883-892

55. Park HY, Park WB, Lee SH et al. Posttraumatic stress disorder and depression of survivors 12 months after the outbreak of Middle East respiratory syndrome in South Korea. BMC Public Health 2020; 20: 605

56. Akalin E, Azzi Y, Bartash R et al. Covid-19 and kidney transplantation. N Engl J Med 2020; 382: 2475-2477

57. Magage S, Lubanda JC, Susa Z et al. Natural history of the respiratory involvement in Anderson-Fabry disease. $J$ Inherit Metab Dis 2007; 30: 790-799
58. Franzen DP, Nowak A, Haile SR et al. Long-term follow-up of pulmonary function in Fabry disease: a bi-center observational study. PLoS One 2017; 12: e0180437

59. Franzen D, Haile SR, Kasper DC et al. Pulmonary involvement in Fabry disease: effect of plasma globotriaosylsphingosine and time to initiation of enzyme replacement therapy. BMJ Open Resp Res 2018; 5: e000277

60. Kai H, Kai M. Interactions of coronaviruses with ACE2, angiotensin II, and RAS inhibitors-lessons from available evidence and insights into COVID-19. Hypertens Res 2020; 43: 648

61. Reynolds HR, Adhikari S, Pulgarin C et al. Renin-angiotensinaldosterone system inhibitors and risk of Covid-19. N Engl J Med 2020; 382: 2441-2448

62. Mancia G, Rea F, Ludergnani M et al. Renin-angiotensin-aldosterone system blockers and the risk of Covid-19. N Engl J Med 2020; 382: 2431-2440

63. European Society of Hypertension, Statement of the European Society of Hypertension (ESH) on hypertension, Renin-Angiotensin System (RAS) blockers and COVID-19 April 15th, 2020. https://professional.heart.org/professional/ ScienceNews/UCM_505836_HFSAACCAHA-statementaddresses-concerns-re-using-RAAS-antagonists-in-COVID19.jsp (13 November 2020, date last accessed)

64. Mehra MR, Desai SS, Kuy S et al. Retraction: cardiovascular disease, drug therapy, and mortality in Covid-19. N Engl J Med 2020; 382: 2582-2582

65. Prabakaran T, Nielsen R, Larsen JV et al. Receptor-mediated endocytosis of alpha-galactosidase $\mathrm{A}$ in human podocytes in Fabry disease. PLoS One 2011; 6: e25065

66. Bonifacino JS, Traub LM. Signals for sorting of transmembrane proteins to endosomes and lysosomes. Annu Rev Biochem 2003; 72: 395-447

67. Belouzard S, Chu VC, Whittaker GR. Activation of the SARS coronavirus spike protein via sequential proteolytic cleavage at two distinct sites. Proc Natl Acad Sci USA 2009; 106: 5871-5876

68. D'Marco L, Puchades MJ, Romero-Parra M et al. Diabetic kidney disease and COVID-19: the crash of two pandemics. Front Med (Lausanne) 2020; 7: 199

69. Dixon DL, Van Tassell BW, Vecchié A et al. Cardiovascular considerations in treating patients with Coronavirus Disease 2019 (COVID-19). J Cardiovasc Pharmacol 2020; 75: 359-367

70. Beigel JH, Tomashek KM et al. Remdesivir for the treatment of COVID-19- Final report. N Engl J Med 2020; doi: 10.1056/ NEJMoa2007764

71. European Medicines Agency. Summary on Compassionate Use of Remdesivir-Gilead. https://www.ema.europa.eu/en/ documents/other/summary-compassionate-use-remdesivirgilead_en.pdf (13 November 2020, date last accessed)

72. Savarino A, Boelaert JR, Cassone A et al. Effects of chloroquine on viral infections: an old drug against today's diseases? Lancet Infect Dis 2003; 3: 722-727

73. Gautret P, Lagier JC, Parola P et al. Hydroxychloroquine and azithromycin as a treatment of COVID-19: results of an open-label non-randomized clinical trial [published online ahead of print. Int J Antimicrob Agents 2020; 56: 105949

74. Molina JM, Delaugerre C, Le Goff J et al. No evidence of rapid antiviral clearance or clinical benefit with the combination of hydroxychloroquine and azithromycin in patients with severe COVID-19 infection. Med Mal Infect 2020; 50: 384

75. Food and Drug Administration. FDA cautions against use of hydroxychloroquine or chloroquine for COVID-19 outside of the hospital setting or a clinical trial due to risk of heart 
rhythm problems. FDA Drug Safety Communication. https:// www.fda.gov/drugs/drug-safety-and-availability/fda-cautionsagainst-use-hydroxychloroquine-or-chloroquine-covid-19outside-hospital-setting-or (24 April 2020, date last accessed)

76. Namdar M. Electrocardiographic changes and arrhythmia in Fabry disease. Front Cardiovasc Med 2016; 3: 7

77. Chorin E, Wadhwani L, Magnani S et al. QT interval prolongation and Torsade De Pointes in patients with COVID-19 treated with hydroxychloroquine/azithromycin [published online ahead of print May 11, 2020]. Heart Rhythm 2020; 17: 1425-1433

78. Trimarchi H. SARS-CoV-2 and Fabry nephropathy: potential risks and the pathophysiological perspective. J Nephropathol 2020; 9: e36

79. Srivatana V, Aggarwal V, Finkelstein FO et al. Peritoneal dialysis for acute kidney injury treatment in the United States: brought to you by the COVID-19 pandemic. Kidney 360 2020; 1: $410-415$

80. Ahn JY, Sohn Y, Lee SH et al. Use of convalescent plasma therapy in two COVID-19 patients with acute respiratory distress syndrome in Korea.J Korean Med Sci 2020; 35: e149

81. Clinicaltrials.gov. Clinical Trial of Nebulized Surfactant for the Treatment of Moderate to Severe COVID-19. https://clinical trials.gov/ct2/show/NCT04362059 (16 October 2020, date last accessed)

82. Germain DP, Elliott P, Falissard B et al. The effect of enzyme replacement therapy on clinical outcomes in male patients with Fabry disease: a systematic literature review by a European panel of experts. Mol Genet Metab Reports 2019; 19: 10045490

83. Wanner C, Arad M, Baron R et al. European expert consensus statement on therapeutic goals in Fabry disease. Mol Genet Metab 2018; 124: 189-203

84. Centers for Disease Control and Prevention (CDC). CDC updates COVID-19 transmission webpage to clarify information about types of spread. Updated May 22, 2020. https://www.cdc.gov/media/releases/2020/s0522-cdc-updatescovid-transmission.html (14 June 2020, date last accessed)

85. Morawska L, Cao J. Airborne transmission of SARS-CoV-2: the world should face the reality. Environ Int 2020; 139: 105730

86. Politei J. Fabry disease during the COVID-19 pandemic. Why and how treatment should be continued. Mol Genet Metab 2020; 130: 227-229
87. Crespo J, Calleja JL, Zapatero A. Widespread covid-19 infection among Spanish healthcare professionals did not occur by chance. BMJ 2020. https://blogs.bmj.com/bmj/2020/06/01/ widespread-covid-19-infection-among-spanish-healthcareprofessionals-did-not-occur-by-chance/ (13 November 2020, date last accessed)

88. International Organization for Standardization. COVID-19 Response: Freely available ISO standards. https://www.iso. org/covid19 (16 October 2020, date last accessed)

89. Chin AWH, Chu JTS, Perera MRA et al. Stability of SARS-CoV2 in different environmental conditions. Lancet Microbe 2020; 1: e10

90. Yuen KS, Ye ZW, Fung SY, Chan CP et al. SARS-CoV-2 and COVID-19: the most important research questions. Cell Biosci 2020; $10: 40$

91. Arons MM, Hatfield KM, Reddy SC et al. Presymptomatic SARS-CoV-2 infections and transmission in a skilled nursing facility. N Engl J Med 2020; 382: 2081-2090

92. Laney DA, Fernhoff PM. Diagnosis of Fabry disease via analysis of family history. J Genet Counsel 2008; 17: 79-83

93. Wang Y, Tian $\mathrm{H}$, Zhang L et al. Reduction of secondary transmission of SARS-CoV-2 in households by face mask use, disinfection and social distancing: a cohort study in Beijing, China. BMJ Glob Health 2020; 5: e002794

94. Fontana F, Giaroni F, Frisina $\mathrm{M}$ et al. SARS-CoV-2 infection in dialysis patients in northern Italy: a single-centre experience. Clin Kidney J 2020; 13: 334-339

95. van der Made CI, Simons A, Schuurs-Hoeijmakers J, et al. Presence of genetic variants among young men with severe COVID-19. JAMA 2020; 324: 663-673

96. Ellinghaus D, Degenhardt F, Bujanda L et al. Genomewide association study of severe Covid-19 with respiratory failure. $N$ Engl J Med 2020; 383: 1522-1534

97. Stawiski EW, Diawnji D, Suryamohan K et al. Human ACE2 receptor polymorphisms predict SARS-CoV-2 susceptibility. bioRxiv 2020; (published ahead of print 10 April 2020; 10.1101/2020.04.07.024752); preprint: not peer reviewed

98. Ballout RA, Sviridov D, Bukrinsky MI et al. The lysosome: a potential juncture between SARS-CoV-2 infectivity and Niemann-Pick disease type $C$, with therapeutic implications. FASEB J 2020; 34: 7253-7264 